\title{
Towards Harmonization IEC61850 and Intelligent Agents: Characteristic Analysis of Service Tracking*
}

\author{
Bin Duan, Cheng Tan, Yan Liu \\ Xiangtan University, Xiangtan, Hunan, China \\ Email: db61850@163.com, tcdreams@126.com,renalee.hi@163.com
}

Received March, 2013

\begin{abstract}
IEC61850 is the most promising standard for design of power utility communication networks and automation systems. On the other hand multi-agent systems are attracting growing interest for different applications of power engineering. This paper presents the possibility that IEC61850 could be applied to build intelligent agents for power engineering applications. The paper investigates the correlation between IEC61850 and multi-agents and presents IEC61850 service tracking as a scenario. First, briefly introduces reporting and service tracking; then, investigates agents, intelligent agents and the characteristics of intelligent agents; last, analyzes the characteristics of service tracking and compares it with intelligent agents. And analysis shows that IEC61850 could be applied to build intelligent agents, which represents a significant step forward for MAS to apply in power engineering.
\end{abstract}

Keywords: Intelligent Agents; Service Tracking; Multi-agent; IEC61850Ed2

\section{Introduction}

IEC61850 is an international standard on communication networks and systems for power utility automation, which has been made by the International Electrotechnical Commission (IEC) technical committee 57. The IEC61850 edition 2 proposes the service tracking mechanism, which is to realize service surveillance and normally applied to the third-party service in new development application. In recent years, study workers from abroad and home have carried out a large number of researches on IEC61850, research fields including power system control [1], protection [2], condition monitoring [3], fault diagnosis [4], and so on.

For over a decade, the proposed use of multi-agent system (MAS) has been reported in many kinds of famous journals and conference papers. MAS technology is now being developed for a range of applications for power Engineering. Four categories of power engineering applications were discovered: monitoring and diagnostics [5], distributed control [6,7], which is the most interesting area, modeling and simulation [8], and protection [9], which is the least active area.

The study of harmonizing is not without precedent: to realize the interoperability goal of smart grid, the Electric Power Research Institute (EPRI) has sponsored the de-

*This research was supported by National Natural Science Foundation of China (NSFC) (No.61170191). Scientific Research Fund of Hunan Provincial Education Department (11CY017) velopment of harmonizing the IEC Common Information Model (CIM) and IEC61850. There is a detail research about the harmonization between the CIM and IEC61850 in [10]. In addition, to investigate the interplay between IEC61850 and IEC61499, there is a deep research on how to combine the application functions of IEC61850-compliant devices with IEC61499-compliant "glue logic" in [11]. Accordingly, we may think that whether can we harmonize IEC61850 and multi-agents, or IEC61850 can be applied to build intelligent agents. If so, it would be very significant for intelligent agents with interoperability to use for power engineering applications. There are more and more discussions on the question that whether if IEC61850 could be applied to build intelligent agents in the world. And some say yes to it, in particular, Karlheinz Schwarz introduced that IEC61850 could be applied to build intelligent agents in his Bokee [12]. But he doesn't make any other research on this problem, such as, why IEC61850 can be used to build intelligent agents? How does IEC61850 used to build intelligent agents? And what is the significance of IEC61850 used to build intelligent agents? This paper is aimed at discussing why IEC61850 can be used to build intelligent agents.

\section{IEC 61850 Reporting and Service Tracking}

\subsection{Reporting}

Reporting meets a number of crucial requirements for 
event-driven information exchange. The data transfer models provide mechanisms for transferring data values caused by well-defined conditions from a logical node to one client [13].

The main characteristics of reporting are:

- Timely reports serve as an indication to clients (optionally keeping sequence-of-events to the client),

- $\quad$ Sending reports only when required (controlled by several attributes),

- Low-frequency integrity scan and client-initiated general interrogation.

Reporting provides mechanisms to report packed values of instances of a data object immediately or after some buffer time.

The principal building blocks and services for reporting is depicted in Figure 1.

The reporting model is composed of three building blocks. Classes are defined for the report control. IEC61850 defines the report-control-block classes for reporting, there are two classes of report control blocks defined: buffered report control block (BRCB) and unbuffered report control block (URCB).

The RCB class definition contains a number of attributions and three services. These attributions can be classified into three kinds: one of which is used to operate the BRCB behavior and represent as the symbol of BRCB, such as RptEna, PurgeBuf, Owner, et al; Another kind is used to control the formation process of report, such as TrgOps, In tgPd, GI, et al; The other kind is used to control the report format, such as SqNum, Entry ID, time of Entry, et al. The three services are report, set RCB Values and get RCB Values.

\subsection{Servicing Tracking}

The reporting and logging function based on the process and function related data model allow to track what happens in the process. For tracking what happens communication wise in the system itself, the IEC61850 edition 2 provides the possibility to track all services, even those with negative responses. For this purpose the service tracking are classified as follows:

- $\quad$ Control block tracking services ;

- $\quad$ Command tracking services;

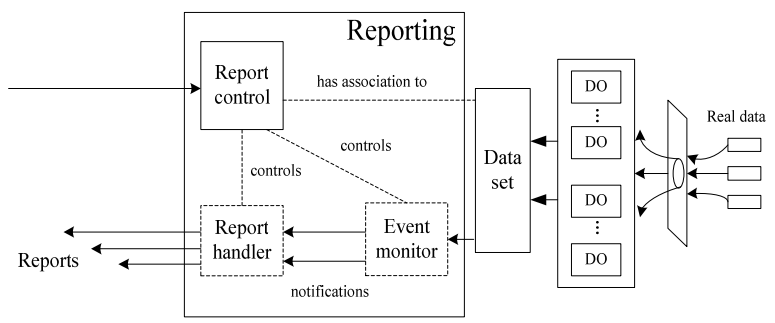

Figure 1. The principal building blocks and services for reporting.
- Other service tracking.

For a given server, there is a single data object instance (tracking data object) available in the object directory for each kind of service, that will mirror the value of the service parameters and its acceptance by the server. This allows that a service can be reported to any client, as soon as the tracking data object is a data-set member of the Dat Set associated to a RCB or to a LCB.

The control block tracking services provide a means to keep track of issued services on or related to the control blocks. Control block value changes may be caused by services setting a specific value or by internal (local) events in the server. The control block tracking service replaces the originally intended use of the FC and TrgOp of the control block attributes of the edition 1 . The tracking service provides the same possibilities in a more consistent way, and with additional features. The model can be used to tracking any service request.

Similarly to the service tracking for control block accesses, the control command can also be tracked. The tracking of control command offers a logistic for tracking any control command applied to a data object.

\section{Intelligent Agents}

\subsection{Overview of Intelligent Agents}

When talking about the concept of an agent, different researchers put forward different ideas and opinions according to their own research background and research field. And so far, there is not a unified definition for an agent. While all the definitions are difference, they all share a basic set of concepts: the notion of an agent, its environment, and the property of autonomy.

From an engineering perspective, this definition is problematic: it does not clearly distinguish agents from a number of existing software and hardware system. In order to help differentiate MAS from existing systems, many researchers extend the concept of an agent. For example, Doctor Wooldridge, the famous agent theory researcher, thinks that an intelligent agent is a computer system which can take a complex autonomous behavior in the specific environment.

\subsection{Characteristics and Functions of Intelligent Agents}

An intelligent agent is a computer entity which can display flexible autonomy in the field of agents, naturally, with the common characteristics of agents. Making a general survey of an agent definition, the characteristics of intelligent agents can be summarized as follows [14]:

- Reactivity: an intelligent agent can react to the change of environment in a timely fashion, and takes some corresponding action according to the change and function which is designed to achieve. 
- Pro-activeness: an intelligent agent can follow the promise, take the initiative and exhibit the goal-directed behavior. Goal-directed behavior connotes that an agent will dynamically change its behavior in order to achieve its goals. For example, if an agent loses communication with another agent whose services it requires to fulfill its goals; it will search for another agent that provides the same services.

- Social ability: an intelligent agent has a certain social ability, that is, intelligent agents are able to communicate with users and resources represented by an agent and other agents. Social ability not only connotes the ability to pass and transmit data between different software and hardware entities, but also to negotiate and interact in a cooperative manner.

Not only does the characteristics of reactivity, pro-activeness, and social ability help us distinguish agents from traditional hardware and software systems, but also we may discuss weather IEC61850 can be applied to build intelligent agents through a comparative analysis of the characteristics between intelligent agents and service tracking.

\section{Characteristic Analysis of Service Tracking}

Reporting mechanism is the information exchange mechanism which is driven by information change. The information contains both the data information in process which also called event information and the configuration parameters of service itself which also called service information. Report is produced when the information change, but only the changed information referenced dataset member values can be reported. The IEC61850 edition 2 defines service tracking which is the development of reporting that is driven by the change of service configuration parameters. The essential difference between reporting and service tracking is that: Reporting is normally applied to report changes of state information and measurement information in process; while service tracking is applied to monitor the behavior between system and equipment or equipment and other equipment in substation.

Service tracking can be implemented with the reporting or logging. Service tracking is only a functionality interface, which must be used through combining with reporting or logging.

Reactivity: Service tracking can adopt the reporting event-driven mechanism, that is, service tracking uses changes of service configuration parameters to drive the reporting. For example, GOCB service tracking trigger mechanism is shown in Figure 2. First, instantiate BRCB, which contain setting BRCB attributions (e.g.

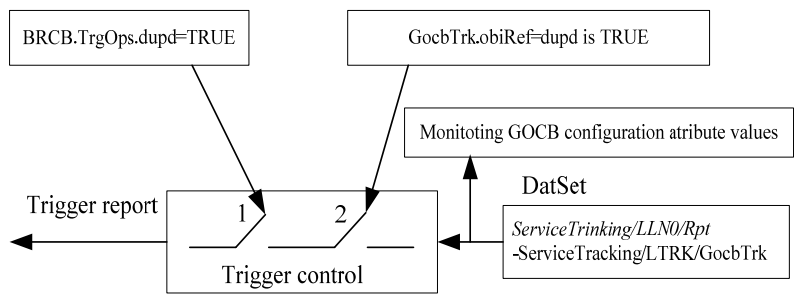

Figure 2. GOCB service tracking trigger mechanism.

BRCB.TrgOps.dupd=TRUE) and setting BRCB DatSet; Second, monitoring GOCB configuration parameter values; Last, triggering report, when one or more values of GOCB configuration parameters changed because of the Set GOCB Values request from a client, the BRCB instance will report all the values of GOCB and CST (common service tracking common data class) configuration parameters at timely. For this, we may think that service tracking has the same reactivity characteristic with intelligent agents.

Pro-activeness: similarly, service tracking can also dynamically change its behavior in order to achieve its goal. For example, the RCB instance sends a report immediately or after some buffer time when the service configuration parameters changed. But in case of the communication association loss between the RCB instance and the client, the RCB instance can not send the service tracking information timely. In order to achieve this goal, service tracking adopts the buffer mechanism of reporting automatically which can be implemented by BRCB. The BRCB instance adds Entry ID, SqNum, Time of Entry to the buffered report, which is convenient to manage the buffered report. Once the communication association is recovery, the BRCB instance will report the new and buffered reports in sequence. In addition, it can also ask Logging for help, because logging has the ability to achieve the goal.

Social ability: Service tracking can also represent the characteristic of social ability. Reporting just transmits the changed data information from one or more logic nodes to a client, but can not confirm the accuracy of the data. For example, when receiving a report after the RCB instance's configuration parameters changed, a client can not parse the message as the report format changed. But service tracking can solve the problems. When one or more RCB instance's configuration parameters change, service tracking will report all values of the RCB instance's configuration parameters to the client, and negotiate with the other software of the client, then decide whether to change the RCB instance's configuration in the client. Through this method, the client can always correctly parse the message transmitted by the RCB instance. Therefore, service tracking can not only pass data, but can cooperate and negotiate with other software.

To sum up the characteristic analysis of service track- 
ing, we know that service tracking may represent the same three characteristics with intelligent agents; therefore, we may think that service tracking could be applied to build intelligent agents.

\section{Conclusions}

This paper opened by posing some questions surrounding IEC61850 and intelligent agents: Why IEC61850 could be applied to build intelligent agents? How does IEC61850 used to build intelligent agents? And what is the significance of IEC61850 used to build intelligent agents? In this paper the first question has been answered, by introducing that what is agents, what is intelligent agents, what characteristics does intelligent agents have and so on. On the other hand, we briefly introduced service tracking and reporting and analyzed the characteristics of service tracking in detail. Through the comparative analysis, we concluded that service tracking could be applied to build intelligent agents.

In order to enhance the benefits of this propose for power engineering applications, we will work on the two main directions to answer the latter two questions in future. First, we are going to use IEC61850 to build a tracking system based on intelligent agents, which is mainly to explore the method of using IEC61850 to build multi-agent system. Second, we will apply the agentsbased tracking system for power engineering applications flexibility, which is about to achieve the significance of applying the agents-based tracking system.

\section{REFERENCES}

[1] D. W. Wang, Y. L. Zhu, J. Di and X. M. Zhai, “A Method for Electric Power Equipment Remote Control Based on IEC 61850," Automation of Electric Power Systems, Vol. 33, No. 5, 2009, pp. 50-54.

[2] M. García-Gracia, S. Borroy, L. Giménez de Urtasun and M. P. Comech, "Novel Protection Scheme Based on IEC61850,” Electric Power Systems Research, Vol. 81, No. 12, 2011, pp. 2178-2187. doi:10.1016/j.epsr.2011.07.017

[3] Y. Ma, J. Y. Zheng, J. Mei and Z. Zhang, "Information Modeling and Communication of SF6 Circuit Breaker On-line Monitoring System Based on IEC61850,” Electric Power Automation Equipment, Vol. 30, No. 2, 2010, pp. 131-134.

[4] D. B. Liu, X. P. Gu and H. P. Li, "Complete Analytic Modeling for Power System Fault Diagnosis Based on IEC61850,” Automation of Electric Power Systems, Vol.
36, No. 10, 2012, pp. 94-100.

[5] E. M. Davidson, S. D. J. McArthur, J. R. McDonald, T. Cumming and I. Watt, "Applying Multi-agent System technology in practice: Automated Management and Analysis of SCADA and Digital Fault Recorder Data," IEEE Transactions on Power Systems, Vol. 21, No. 2, 2006, pp. 559-567. doi:10.1109/TPWRS.2006.873109

[6] I. S. Baxevanos and D. P. Labridis, "Implementing Multi Agent Systems Technology for Power Distribution Network Control and Protection Management,” IEEE Transactions on Power Del., Vol. 22, No. 1, 2007, pp. 433-443. doi:10.1109/TPWRD.2006.877085

[7] C.-X. Dou, S.-J. Jin, G.-T. Jiang and Z.-Q. Bo, "Multi-Agent Based Control Framework for Microgrids," in 2009 Asia-Pacific Power and Energy Engineering Conference, Wuhan, China, 2009, pp. 1-4.

[8] T. Li, Z. Xiao, M. Huang, J. Yu and J. Hu, "Control System Simulation of Microgrid Based on IP and Multi-Agent," in 2010 International Conference on Information, Networking and Automation (ICINA), Kunming, China, 2010, pp. 235 -239. doi:10.1109/ICISA.2010.5480311

[9] I. Zabet and M. Montazeri, "Implementing Cooperative Agent-based Protection and Outage Management System for Power Distribution Network Control,” The 4th International Power Engineering and Optimization Conf. (PEOCO2010), Shah Alam, Selangor, MALAYSIA: 23-24 June 2010, pp. 318-324.

[10] Z. Y. Gao, J. G. Yao, S. C. Yang, et al., "Survey of Coordination Scheme between CIM and IEC61850 Model," Automation of Electric Power Systems, Vol. 35, No. 16, 2011, pp. 9-14.

[11] V. Vyatkin, G. Zhabelova, N. Higgins, K. Schwarz and N. K. C. Nair, "Towards Intelligent Smart Grid Devices with IEC 61850 Interoperability and IEC 61499 Open Control Architecture,"

[12] K. Schwarz, Can IEC 61850-7-2 Edition 2, be used to build Agents [EB/OL] [2011-07-12]. http://blog.iec61850.com/2011/07/can-iec-61850-7-2-edit ion-2-be-used-to.html

[13] IEC61850-7-2 Ed. 2, CDV Basic Information and Communication Structure: Abstract Communication Service Interface (ASCI).

[14] S. D. J. Mcarthur, E. M. Davidson, V. M. Catterson, A. L. Dimeas, N. D. Hatziargyriou, F. Ponci and T. Funabashi, "Multi-agent Systems for Power Engineering Application Part I: Concepts, Approaches, and Technical Challenges," IEEE Transactions on Power Systems, Vol. 22, No. 4, 2007, pp. 1743-1752.doi:10.1109/TPWRS.2007.908471 